\title{
PLANNING UAV-ASSISTED VISUAL INSPECTIONS OF CONSTRUCTION SITES
}

\author{
Henk Freimuth ${ }^{1}$, Jan Müller² ${ }^{2}$ and Markus König ${ }^{3}$
}

\begin{abstract}
Inspection flights on construction sites, carried out with Unmanned Aerial Systems (UASs), should be planned with safety as the primary priority. Although the generation of waypoints may be conducted in an automated fashion, with distanceto-object constraints implemented in the algorithm, the visual review of such flight paths by a human operator before take-off is crucial. Apart from safety concerns, finding the right camera orientation for taking photographs of specific details is a non-trivial task when the camera is mounted to an Unmanned Aerial Vehicle (UAV). The inspection planning application should interactively assist the operator with this task.

We propose an interactive and simulation-based inspection flight planning concept. A realistic and detailed simulation allows a quick and intuitive insight about generated flight paths and possible shortcomings of such paths in terms of flight safety. Images, rendered from the virtual scene, with the flying camera's point of view enable the operator to assess the quality of the intended photographs before conducting the flight mission. These previews take into account the main parameters of the camera such as focal length and natural lighting. This minimises the need to readjust parameters or repeat inspection flights to get the right picture.
\end{abstract}

Keywords: Visual Inspection, Unmanned Aerial Systems, 4D Building Information Modeling, Progress Monitoring, Ground Control, Path Planning.

\section{INTRODUCTION}

The full benefit of Building Information Modelling (BIM) may only be harnessed by minimising the discrepancy between as-planned and as-built information on a daily basis (Bosché et al. 2014). The development of an efficient workflow for gathering data of the construction site is crucial to achieving a steady correlation between the ever-evolving model data and current changes in the real world. In contrast to walking around the site and repeatedly taking photos of the same places, using Unmanned Aerial Systems (UASs) for progress monitoring and inspection tasks on construction sites promises an efficient method for collecting such data. The idea of creating a method for providing data for coordination tasks is inspired by the fact that the flight missions for a UAV can also be based on the spatial and meta information in the model data. The ability to derive the location and process information from a building information model for a certain task and using these as a trigger for capturing imagery of the newly built structure, for example, the installation of a window, lays the theoretical foundation for an integrated use of UAS on construction sites.

1 Research Associate, Chair of Computing in Engineering, Faculty of Civil and Environmental Engineering, Ruhr-University Bochum, Germany, henk.freimuth@rub.de

2 Research Assistant, Chair of Computing in Engineering, Faculty of Civil and Environmental Engineering, Ruhr-University Bochum, Germany, jan.müller@rub.de

3 Head of Institute, Chair of Computing in Engineering, Faculty of Civil and Environmental Engineering, Ruhr-University Bochum, Germany, koenig@inf.bi.rub.de 
However, the technical implementation must adhere to the capabilities of current UAS technology. Infallible obstacle avoidance is the key challenge for safe use of UAVs no matter the intended use. Yet, recent commercial solutions may still overlook or misinterpret their environment which requires the operator to be able to take control at all times. For inspection tasks, operators rely on handheld remote controls for manual control of the UAS. For advanced inspection tasks, covering large areas or complex surroundings, the operator is best advised to prepare autonomous flight missions in a ground station application. Such ground station applications follow a common design principle: a two-dimensional map view with annotations visualising waypoints and flight paths of the UAV and a dot shape depicting the current position of the UAV in flight. Depending on the qualitative requirements towards the resulting imagery, a second operator is often required for live camera control. A live view of the camera's point of view is then transferred to the second operator's screen so he may steadily adjust the camera system's parameters.

These observations lead to the conclusion that the planning and execution phases for inspections with UAS may be greatly optimised in terms of efficiency and safety. In the planning stage of an inspection a ground station application should convey all relevant information to the user and it should implement a set of rules that unburden the user from manually measuring safe distances to physical objects. Furthermore, the application should assist the planning personnel to visually assess the point of interest by virtually generating photographs in the three-dimensional scene.

As a result, the main contribution of this work is the proposal of an advanced inspection solution, implemented by a prototypical ground station application to allow the assessment of the practicability and eventual shortcomings. The application features a communication interface with the established Dronecode (2015) ecosystem including a Software-In-The-Loop simulation for realistic simulation of inspections, a threedimensional user interface that runs in common browsers, exemplary waypoint generation for points of interest as well as pre-rendered photo perspectives that convey a realistic impression of the expected resulting imagery.

\section{RELATED WORK}

UAS have been adopted for various applications ranging from documentation of excavation sites in archaeology (Fallavollita et al. 2013), crop analysis in Precision Agriculture (Peña et al. 2013) with commercial solutions readily available (Nixon 2016), and emergency tasks such as the investigation of forest fires with multiple aircraft or reconnaissance of areas damaged by floods or earthquakes (Casbeer et al. 2006; Baiocchi et al. 2013). The ability to quickly deploy a lightweight aircraft and gain oversight of an otherwise incomprehensible situation has proven UAS to be a utility of real worth. Furthermore, the robotic properties of these aircraft promise more accurate sensing, mostly due to the exact repeatability of flight movements and evolving autonomous features.

Han et al. (2015) present a study of how to efficiently combine the advantages of collecting data with UASs and structuring it with 4D BIM technology. They propose a web-based application that allows users to conduct analytical and communicative tasks on both plan- and as-built data. The work is mainly targeted at joining 3D/4D point clouds with plan models, but addresses some common issues when using UASs on construction sites. One of those issues addressed is that common ground control station applications do not suffice as path planning tools. The two-dimensional views and static map data leave 
out important information about the spatial extent of physical objects which can become a safety hazard. Furthermore, this common way of planning flight missions does not allow recognition of occlusions.

Lin et al. (2015) discuss the potential of replacing traditional site photography with a model-driven acquisition of imagery and consecutive, systematic joining of images and $4 \mathrm{D}$ BIM. The authors recognise the quality of images collected by the traditional method varies heavily in terms of location and field of view, demanding an experienced site inspector. However, regarding the use of UAVs on construction sites, the authors argue the practicability of existing, popular UAS platforms. Furthermore, they doubt the applicability of GPS-based navigation methods for the use on densely crowded construction sites in urban environments. In regards to collecting and using aerial imagery for BIM-driven projects, the authors find that the analysis of imagery with BIM is already automated to some degree. However, the collection of these images and the automation of this task follows no strategy as yet.

Hallermann et al. (2014) discuss different flight strategies for obtaining aerial imagery with the purpose of monitoring, one of which is named "Point of Interest". A circular flight path was generated around a PoI and each waypoint triggered the camera with a given attitude towards the PoI. This approach is easily applicable on simple structures such as chimneys, but finding the right camera angles for more complex structures is not necessarily trivial. However, the idea of finding different perspectives to a PoI is comparable to the new feature of the ground control station proposed in this work.

The assessment of these works shows that although the concept of employing UAVs for inspection and progress monitoring purposes is actively being worked on and the industry is already using it to some degree the structured coordination of UAV flights based on 4D BIM data is yet to be explored.

\section{Research Methodology and CONCEPTUAL DESIGN}

This work proposes an enhanced workflow for collecting as-built data from construction sites for the use in 4D BIM, realised by the development of a prototypical application. The application demonstrates improvements both in terms of assisting operating personnel in generating sane flight paths and effectively using 4D BIM as a means for transferring physical objects into a virtual environment.

One of the main goals of the proposed application is to enable site inspectors to generate safe flight paths in an intuitive way. Fig. 1 shows a schematic overview of the components and the interaction flow. The first interaction is the selection of a BIM project to work on. The implementation of an inspection planning application for construction sites relies heavily on BIM. Geometries of building structures in BIM models are the foundation for computing spaces safe for flight on construction sites. The geodetic reference of the site entity makes it possible to project Cartesian coordinates to geolocations in WGS84 notation, which is the coordinate system the UAV navigates in. Completeness of the model in terms of objects that occupy space and may pose a risk for collisions is a substantial pre-requisite for this concept. The consideration of schedules and dynamic site layouts enables time-dependent path planning. The relevant BIM data can be imported by using the open source BIMserver (Beetz et al. 2010). 


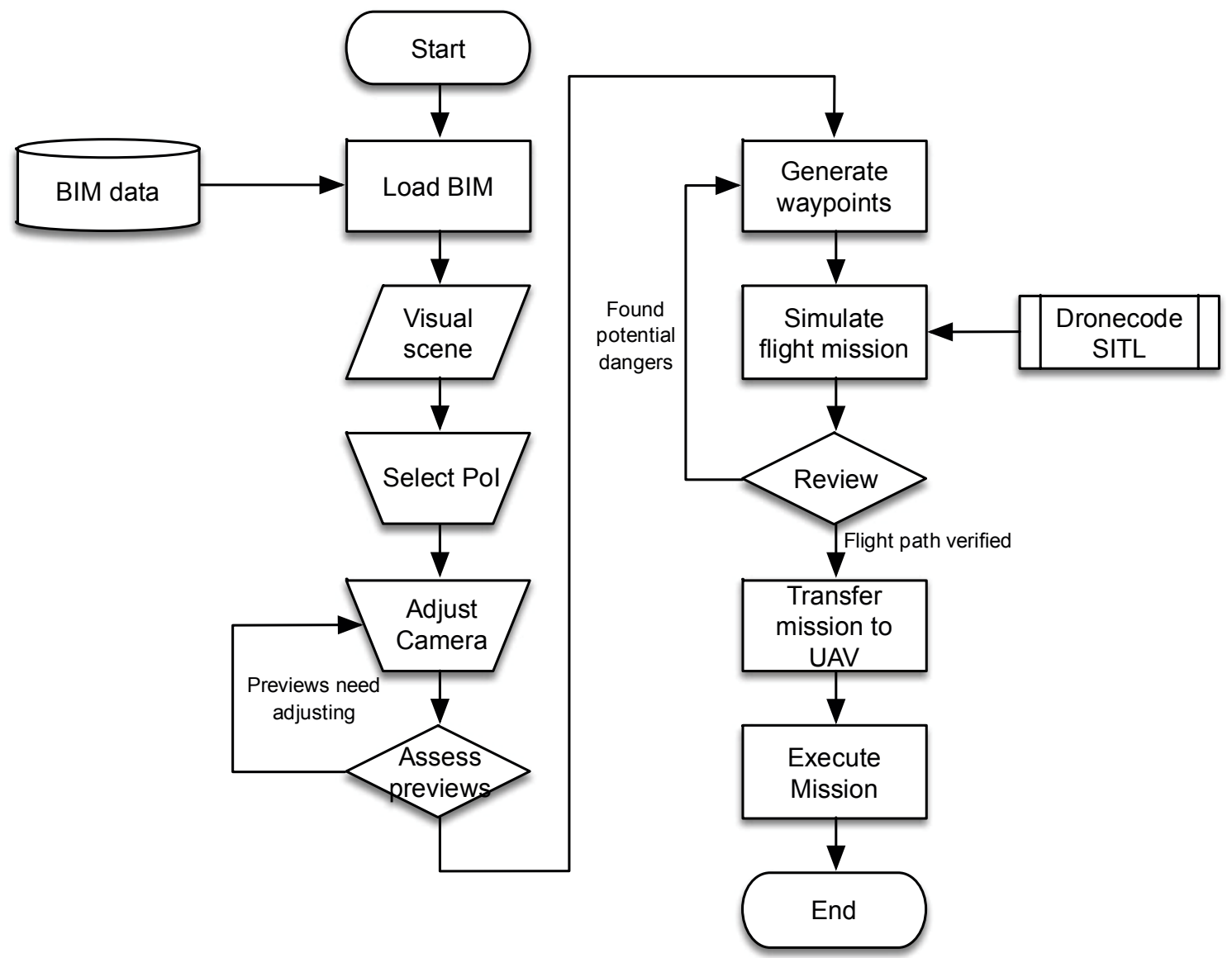

Figure 1: The flow chart depicting user interactions

After importing the user may place and evaluate one or more points of interest. After the selection of points of interest, the application will trigger the path planning algorithm (Freimuth and König 2015). The optimal flight path is an aggregate of different components.

- The take-off location of the UAV, which is either placed manually by the operator in the planning stage or determined implicitly from the reported location of the actual UAV before initiating the inspection flight.

- A sequence of waypoints between the take-off location and the points of interest. Following the path guides the UAV through a computed safe space, abiding the aforementioned ruleset.

- The points of interest, which themselves are associated with additional waypoints.

The path planning concept is separated into three steps: segmentation of the scene, graph generation and invocation of common methods for solving shortest path problems.

For the segmentation step the concept of navigation meshes, established in robotics research, was adapted. Navigation meshes are typically an aggregate of 2D polygons that represent the areas of a robot's environment that are accessible. However, with UAV navigation a $3 \mathrm{D}$ concept is necessary, allowing the UAV to travel in the vertical dimension and to account for variable height of physical objects. The navigation volume is represented as a set of voxels, where no intersections with relevant geometries from the BIM occur. Each voxel inherits information about its neighbourhood of voxels and the potential waypoint it represents. 
Based on the relations between voxels, a graph is generated, with each transition between nodes representing a possible movement of the UAV from one waypoint to the corresponding one of a neighbouring voxel.

Provided start and end points a shortest path algorithm is invoked. By applying penalties in the form of increased transition costs for vertical travel, the shortest path algorithm favours lateral avoidance strategies to climbing over obstacles. This benefits the legislative criterion requiring the operator to keep the UAV in line-of-sight at all times. Further modifications of this kind may be applied in further development stages of a practical path-finding strategy.

After choosing the points of interest, the user is offered the chance to review and confirm the generated flight path. The review process involves simulating the aircraft flying along the flight path, allowing the user to comprehend the motion dynamics of the operation. Therefore, instead of implementing the simulation stage as a simple translation of the UAV model from an arbitrary point a to point $b$, the actual flight behaviour of the target control system Dronecode (2015) was invoked. Given the Software-In-The-Loop (SITL) simulation capabilities of the open source UAS platform chosen for this project, it opens up the possibility of simulating and animating all actions of the aircraft with realistic control dynamics. The simulation furthermore implies a set of real world parameters to be interpreted by the virtually executed firmware. As an example, lowering the number of satellites perceived by the virtual GNSS sensor would result in a potentially uncontrollable or inaccurate flight behaviour, which in turn reveals the possibility to simulate a multitude of inhibiting factors and thus the ability to verify flight missions against different problem scenarios.

After simulating, reviewing, and verifying the flight mission the user may transfer the waypoint and camera control data to the UAV and initiate the autonomous mission. The application will continue monitoring the UAV's behaviour in the real world, as is common among ground station applications, and the user may fully concentrate on the UAV, capable of instantly intervening with manual controls in case of failure, as required, for instance, by law in Germany (see BMVI 2014).

The implementation of the ground control station application widely differs from other established software in this field. Not only is its user interface designed around a 3D scene, it's also divided into a client-server architecture.

The client-server architecture was chosen to enable a sensible delinking of the program logic (for example, the BIM interface or the UAV/simulation link) and the user interface with its analytic 3D scene. Furthermore, this architecture allows the application to be used in different deployment scenarios, two of which are shown in Fig. 2. While planning the inspection, the application may be used in a desktop environment (Fig. 2, right). In the second deployment scenario the application is used on the construction site (Fig. 2, left). The UAV is equipped with a highly portable, low-cost single board computer (SBC) such as the Raspberry Pi. The SBC runs the same inspection planner application as the server, the only difference being that its UAV interface connects to the microcontroller flight control via a serial link instead of establishing a networked link with the simulation framework. 


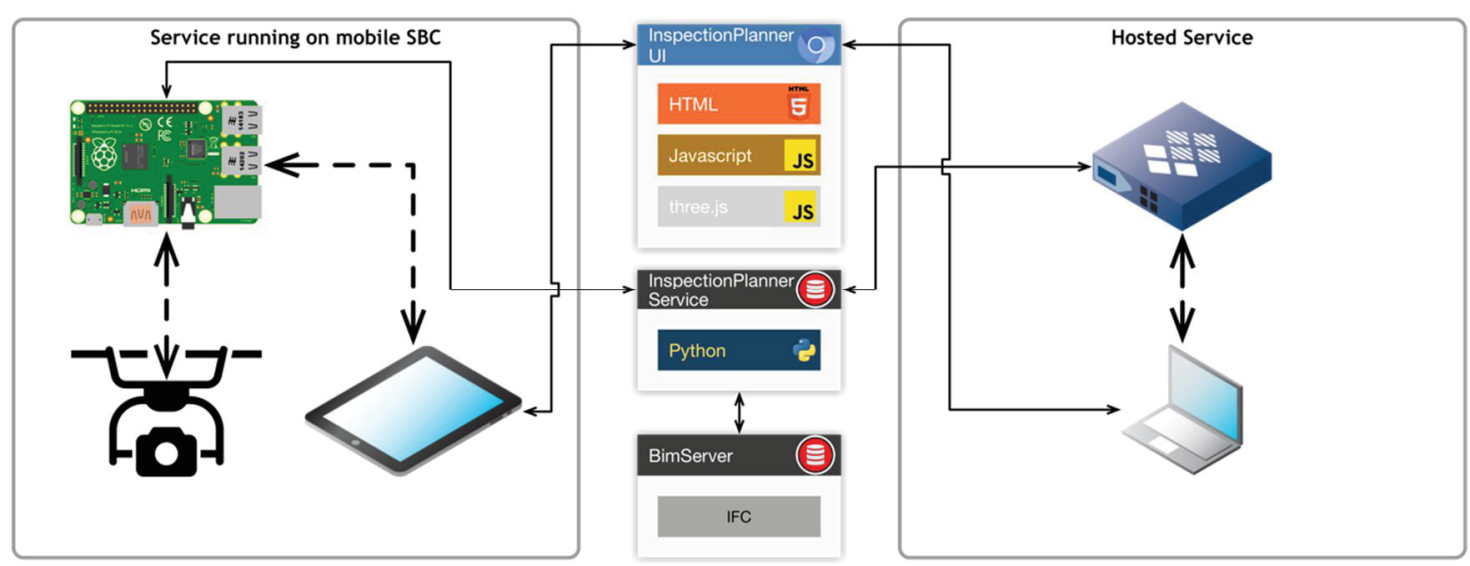

Figure 2: Two different deployment scenarios for the software architecture.

The client device in this scenario is pictured as a tablet device, which is ideally mounted to the remote control. Since the operator was able to thoroughly plan the inspection in a calm environment, he can now pay full attention to the flying aircraft with the tablet device monitoring all important telemetry data, mirroring the flight in the 3D scene.

\section{CASE STUDY}

A case study on the newly rebuilt SSC building on the campus of the Ruhr-University Bochum was carried out for validating the proposed solution. The building information model, created in the course of this study, exhibits a sufficient number of structural elements to conduct planning and simulation of inspections. Fig. 3 shows a brief view of the model compared to a photo of the actual building.
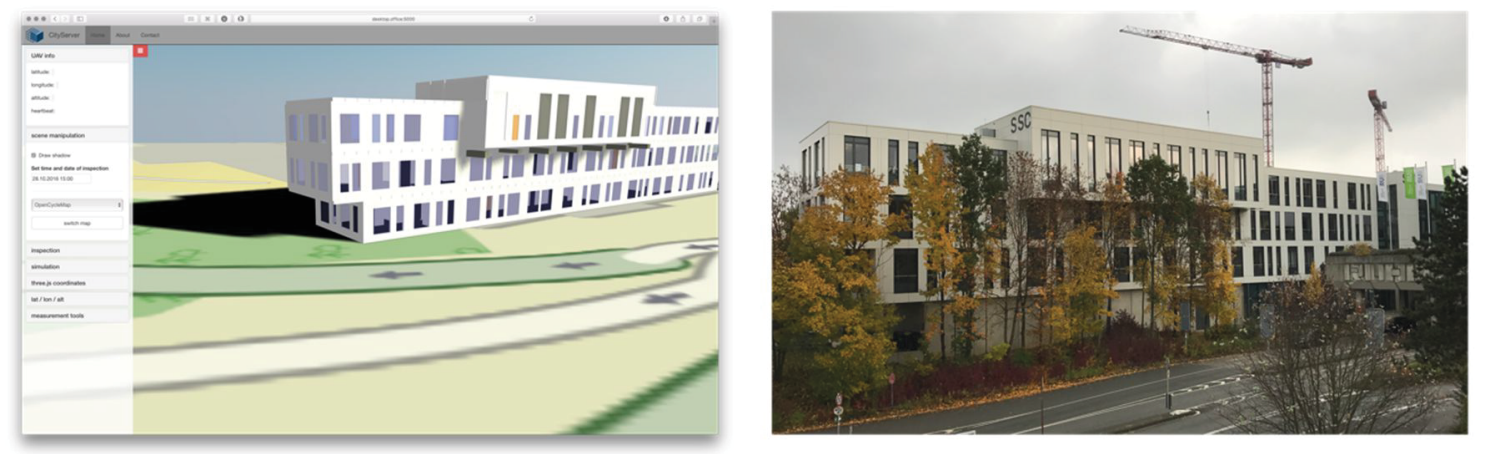

Figure 3: The model juxtaposed with a photograph of the real structure.

The model contains a manually defined geodetic reference sufficient for testing purposes. The building is placed on a ground plane with map tiles from OpenStreetMap to enable higher contextual awareness of the surroundings of the construction site. Although it may not be included in the model data, a dense urban environment around the site may pose inhibiting factors to inspection flights, such as nearby highway lanes or public spaces with crowds of people. The projection of a map plane conveys a quick understanding of such potential problems. The 3D scene is maneuverable in an intuitive manner and the collapsible panel on the left side houses all control- and information elements, which are relevant to creating a flight path.

Fig. 4 shows an example of the simulated UAV flying along a sequence of waypoints that guide the aircraft to a designated PoI. The user activates the respective tool from the 
side panel and may then click any arbitrary point in the scene to create a PoI at that location. Clicking on a façade structure places a point directly on the surface of the façade. Since this newly created point would not be suitable as a waypoint for the UAV, the application generates another point in front of the façade. The placement of such new points may be determined by a ruleset that, among others, defines minimum distance guidelines between UAV and different types of physical objects. A detailed definition of such a ruleset should be derived from empirical knowledge, for instance, the influence of wind on the aircraft, and deterministic facts, such as the expected accuracy of GNSS sensors. To further the idea of collecting valuable imagery of a PoI, instead of computing only one location for taking a photo, the application will find additional vantage points for the UAV. Each of the vantage points is again verified to not violate a safety rule and implies the accurate location for the UAV as well as individual parameters for orienting the stabilised camera system towards the originally selected PoI. The ability to have multiple different shots taken automatically while only selecting one particular point offers improved insight for the inspector, as only one of those vantage points may reveal an unexpected detail in later analysis. Regarding the workflow, at this stage the operator is presented with the option to review pre-rendered photos (Fig. 4b) of the individual vantage points and eventually discard them or relocate the PoI to find a better suited array of vantage points.

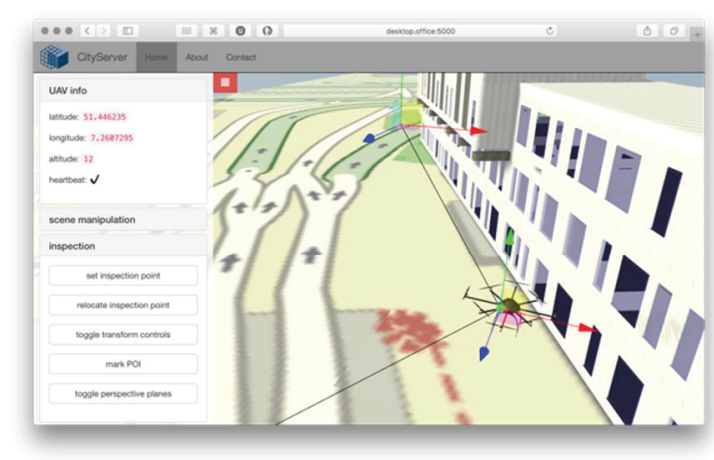

(a)

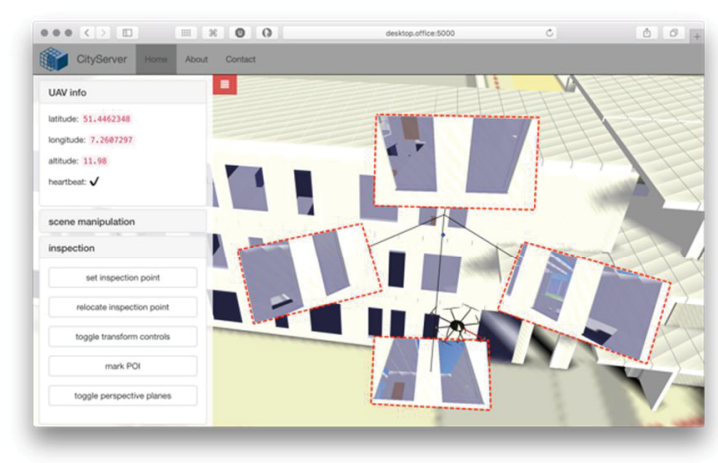

(b)

Figure 4: A simulated UAV hovering at a user-defined waypoint (a) and an exemplary PoI with preview images of the expected photographs (b).

\section{CONCLUSIONS AND OUTLOOK}

This work presents a practical approach to contribute to ongoing research on progress monitoring and visual inspections of buildings, conducted with UAVs. The combination of rich BIM information and UAV navigation methods, the client-server architecture for multiple use cases, and the 3D planning interface are the main contributions. The application is still at an early development stage and apart from investigating the following ideas, further development will focus on tapping the full potential of the SITL simulation method.

The integration of Digital Elevation Models (DEMs) promises more accurate planning and may be continuously updated by the UAS with photogrammetry methods. Extending the simulation stage of the application with a first-person flying mode might convey an even better understanding of the planned flight to the operating personnel. The continuity and integration of 4D BIM concepts can be improved by allowing the user to pick specific structural elements and to filter elements that changed in a certain timespan. With 
timespans and structural differences available, the association of those elements with aerial imagery is a logical step for reaching a structured documentation workflow.

\section{REFERENCES}

Baiocchi, V., Dominici, D. and Mormile, M., 2013. Unmanned aerial vehicle for post seismic and other hazard scenarios. Wit Transactions on the Built Environment, 134, pp.113-122.

Beetz, J., van Berlo, L., de Laat, R., and van den Helm, P. (2010). bimserver. org-An Open Source IFC Model Server. In Proc. CIB W78 2010: 27th International ConferenceCairo, Egypt, (Weise 2006), 16-18.

Bundesministerium für Verkehr und digitale Infrastruktur, BMVI (2014), Kurzinformation über die Nutzung von unbemannten Luftfahrtsystemen. Available at: http://www.bmvi.de/SharedDocs/DE/Publikationen/LF/unbemannteluftfahrtsysteme.html [Accessed 24 Oct. 2016].

Bosché, F., Ahmed, M., Turkan, Y., Haas, C. T., and Haas, R. (2014). The value of integrating Scan-to-BIM and Scan-vs-BIM techniques for construction monitoring using laser scanning and BIM: The case of cylindrical MEP components. Automation in Construction, 49, 201- 213.

Casbeer, D.W., Kingston, D.B., Beard, R.W. and McLain, T.W., 2006. Cooperative forest fire surveillance using a team of small unmanned air vehicles. International Journal of Systems Science, 37(6), pp.351-360.

Dronecode Project, Inc., Dronecode Project: A Linux Foundation Collaborative Project. (2015). Available at: https://www.dronecode.org/about [Accessed 26 Oct. 2016].

Fallavollita, P., Balsi, M., Esposito, S., Melis, M.G., Milanese, M. and Zappino, L., 2013. Uas for archaeology. new perspectives on aerial documentation. ISPRS-International Archives of the Photogrammetry, Remote Sensing and Spatial Information Sciences, 1(2), pp.131-135.

Freimuth, H., König, M. (2015). Generation of Waypoints for UAV-Assisted Progress Monitoring and Acceptance of Construction Work, $15^{\text {th }}$ International Conference on Construction Applications of Virtual Reality (CONVR), pp. 77-86.

Hallermann N., Morgenthal G. (2014). Visual inspection strategies for large bridges using unmanned aerial vehicles (uav), th IABMAS, International Conference on Bridge Maintenance, Safety and Management, Shanghai.

Han, K., Lin, J., \& Golparvar-Fard, M. (2015). A formalism for utilization of autonomous vision-based systems and integrated project models for construction progress monitoring. In Proc., 2015 Conference on Autonomous and Robotic Construction of Infrastructure.

Lin, JJ., Han. K. and Golparvar-Fard, M. (2015). A Framework for Model-driven Acquisition and Analytics of Visual Data using UAVs for Automated Construction Progress Monitoring. 2015 International Workshop on Computing in Civil Engineering, ASCE, Austin, TX, 2015, 156-164.

Nixon, A. (2016). How To Buy an Agriculture Drone: An In-Depth Buyer's Guide. Available at: http://bestdroneforthejob.com/drone-buying-guides/agriculture-dronebuyers-guide/ [Accessed 31 Oct 2016].

Peña, J.M., Torres-Sánchez, J., de Castro, A.I., Kelly, M. and López-Granados, F., (2013). Weed mapping in early-season maize fields using object-based analysis of unmanned aerial vehicle (UAV) images. PLoS One, 8(10), p.e77151. 